\title{
DIFFERENTIAL POLYNOMIALS AND DECAY AT INFINITY
}

\author{
BY FRANÇOIS TRÈVES \\ Communicated by Gerhard Hochschild, January 17, 1960
}

Let $T$ be an element of some space $\mathfrak{F}$ of distributions on $R^{n}$. For which partial differential operators $P(D)$ with constant coefficients, may we claim that

(P) if the support of $P(D) T$ is compact, so is the support of $T$ itself?

To get a reasonable answer to such a question, we must clearly impose, on the elements of $\mathfrak{F}$, conditions of decay at infinity. We give here a classification of all differential polynomials, with respect to property $(\mathrm{P})$, when $\mathcal{T}$ is the space of distributions rapidly decreasing at infinity (see below).

Observe that $(\mathrm{P})$ holds true for any differential polynomial when $\mathfrak{H C}$ is the space of tempered distributions $u$ whose Fourier transforms $\hat{u}$ can be continued to $C^{n}$ as entire functions. For, to say that $P(D) u$ has compact support is the same as to say that $P(y) \mathfrak{u}(y)$ is an entire function of exponential type. But then, $\hat{u}(y)$, being already entire, must be of exponential type (Malgrange [1, Chapter II]) and therefore $u(x)$ must have compact support.

We denote by $z$ the variable in the complex space $C^{n}: z=\left(z_{1}, \cdots, z_{n}\right)$; the real space $R^{n}$ will be canonically imbedded in $C^{n}$ : a point $z$ of $C^{n}$ belongs to $R^{n}$ if all the components $z_{j}$ are reals; in this case, it is called real and denoted by $x=\left(x_{1}, \cdots, x_{n}\right)$ or $y=\left(y_{1}, \cdots, y_{n}\right)$. We consider a polynomial $P(z)$ on $C^{n}$, to which we associate the differential polynomial $P(D)$ on $R^{n}$ obtained by substituting $\partial / \partial x_{j}$ for $2 i \pi z_{j}(1 \leqq j \leqq n)$.

Let us factorize $P(z)$ in irreducible factors:

$$
P(z)=P_{1}(z) \cdots P_{r}(z) .
$$

For a polynomial $Q(z)$ we consider the following property:

(A) The variety of zeros of $Q(z)$ in $C^{n}$ intersects $R^{n}$.

Definition 1. We shall say that $P(z)$ is of type $\mathrm{I}$ if all its irreducible factors have property (A), of type II if at least one of its irreducible factors does not have property (A), of type III if none of them has property (A).

We shall make use, now, of the spaces $D, s, \mathcal{E}^{\prime}$; for their definitions and properties, we refer to Schwartz [1]. We shall also need the space $O_{C}^{\prime}$ : it is the space of distributions rapidly decreasing at infinity, or, in other words, the space of tempered distributions whose Fourier transforms are $C^{\infty}$ functions having, as well as all their derivatives, 
polynomial growth at infinity. Following Schwartz, we denote by $O_{M}$ the space of Fourier transforms of distributions belonging to $O_{C}^{\prime}$.

Notice that if $P(z)$ is of type III, it is never zero on $R^{n}$; therefore, $1 / P(x)$ belongs to $O_{M}$ and its Fourier transform $E$ belongs to $O_{C}^{\prime}$. Obviously, $P(D) E=\delta$. Conversely, if there is a distribution $E \in O_{C}^{\prime}$ such that $P(D) E=\delta$, its Fourier transform $\hat{E}(y)$ is a $C^{\infty}$ function on $R^{n}$ and satisfies $P(y) E(y)=1$, which implies that $P(y)$ can never be zero on $R^{n}$.

Thus: for the polynomial $P(z)$ to be of type III, it is necessary and sufficient that the differential polynomial $P(D)$ has a fundamental solution $E$ belonging to $O_{C}^{\prime}$.

THEOREM 1. The following properties are equivalent:

(a) $P(z)$ is of type I;

(b) for any distribution $T \in O_{C}^{\prime}, P(D) T \in \mathcal{E}^{\prime}$ implies $T \in \mathcal{E}^{\prime}$;

(c) for any function $\alpha(x) \in \mathcal{S}, P(D) \alpha(x) \in D$ implies $\alpha(x) \in D$.

(b) $\Rightarrow$ (c) is trivial. Assume that $P(z)$ is not of type I. Then $P(z)$ $=Q(z) R(z)$, where $R(z)$ is of type III (and of degree $>0$ ). We know then that there is a distribution $E \in O_{C}^{\prime}$ such that $R(D) E=\delta$; of course, $E \notin \mathcal{E}^{\prime}$. Let $\beta(x)$ be any function belonging to $D ; \beta(x) * E \in \mathcal{S}$ and one can choose $\beta$ in such a way that $\beta * E \notin D$; but $P(D)(\beta * E)$ $=Q(D) \beta \in D$; hence, $(\mathrm{c}) \Rightarrow(\mathrm{a})$. We have to prove that $(\mathrm{a}) \Rightarrow(\mathrm{b})$.

We may as well suppose $P(z)$ irreducible; the variety of zeros of $P(z)$ intersects the real space. Let $T \in O_{C}^{\prime}$ be such that $S=P(D) T$ has compact support. We denote by $u(y)$ (resp. $v(y)$ ) the Fourier transform of $T$ (resp. $S$ ). From Paley-Wiener's theorem follows that $v(y)$ can be continued to $C^{n}$ as an entire function $v(z)$ of exponential type. We have, for any real $y, P(y) u(y)=v(y)$. From this we derive that $u(y)$ can be continued, in some neighbourhood of every real point $y$, as an holomorphic function. Therefore the meromorphic function $v(z) / P(z)$ is holomorphic in some open set intersecting the variety of zeros of $P(z)$. But $P(z)$, being algebraically irreducible, is analytically irreducible; and hence the polar locus of $v(z) / P(z)$ must be empty. That means that $u(y)$ can be continued as an entire function $u(z)$ to $C^{n}$, necessarily of exponential type, since it is the quotient of two entire functions of exponential type.

If $P(z)$ is of type $\mathrm{I}$ and if, for some $T \in O_{C}^{\prime}, P(D) T$ has compact support, then, not only has $T$ compact support, but the convex hull of the support of $T$ and the convex hull of the support of $P(D) T$ are the same. This is a trivial consequence of the theorem of supports (Lions [1]).

Of course, we have obtained a necessary and sufficient condition 
to be of type II: $P(z)$ is of type II if there exists a distribution $F \in O_{C}^{\prime}$ such that $P(D) F$ has its support at the origin, without this being true for $F$ itself.

THEOREM 2. $P(z)$ is of type I in each of the following cases:

(1) $P(z)$ is a homogeneous polynomial of degree $>0$;

(2) $P(z)$ is a polynomial in $z_{1}+i z_{2}, \cdots, z_{r}+i z_{2 r}(2 r \leqq n)$.

If $P(z)$ has any of these two properties, so do its irreducible factors and hence, we have only to prove that they imply that the variety of zeros of $P(z)$ intersects the real space. It is trivial for (1); for (2), we observe that, if $z^{*} \in C^{n}$ is a zero of $P(z)$, this is also true of

$$
\left(x_{1}^{*}-y_{r+1}^{*}, \cdots, x_{r}^{*}-y_{2 r}^{*}, y_{1}^{*}+x_{r+1}^{*}, \cdots, y_{r}^{*}+x_{2 r}^{*}\right) \text {, }
$$

where the $x_{j}^{*}$ and $y_{j}^{*}$ are real and $x_{j}^{*}+i y_{j}^{*}=z_{j}^{*}(1 \leqq j \leqq r)$.

\section{REFERENCES}

J. L. LIONS

1. Supports dans la transformation de Laplace, J. Analyse Math. vol. 2 (1953) pp. 369-380.

B. Malgrange

1. Existence et approximation des solutions des équations aux dérivées partielles et des équations de convolution, Ann. Inst. Fourier, Grenoble vol. 6 (1955-1956) pp. 271-355.

L. SCHWARTZ

1. Theorie des distributions, Paris, Hermann, 1957-1958.

University of California, Berkeley 